\title{
Optimierung von FSS-Bandpassfiltern mit Hilfe der Schwarmintelligenz (Particle Swarm Optimization)
}

\author{
G. $\mathbf{W u}^{1}$, V. Hansen ${ }^{1}$, E. Kreysa ${ }^{2}$, and H.-P. Gemünd ${ }^{2}$ \\ ${ }^{1}$ Lehrstuhl für Theoretische Elektrotechnik, Bergische Universität Wuppertal, Germany \\ ${ }^{2}$ Max-Planck-Institut für Radioastronomie, Bonn, Germany
}

Zusammenfassung. In diesem Beitrag wird ein neues Verfahren zur Optimierung von Bandpassfiltern aus mehrlagigen frequenzselektiven Schirmen (FSS), die in ein Dielektrikum eingebettet sind, vorgestellt. Das Ziel ist es, die Parameter der gesamten Struktur so zu optimieren, dass ihre Transmissionseigenschaften hohe Filteranforderungen erfüllen. Als Optimierungsverfahren wird die Particle Swarm Optimization (PSO) eingesetzt. PSO ist eine neue stochastische Optimierungsmethode, die in verschieden Gebieten, besonders aber bei der Optimierung nicht linearer Probleme mit mehreren Zielfunktionen erfolgreich eingesetzt wird. In dieser Arbeit wird die PSO in die Spektralbereichsanalyse zur Berechnung komplexer FSS-Strukturen integriert. Die numerische Berechnung basiert auf einer Integralgleichungsformulierung mit Hilfe der spektralen Greenschen Funktion für geschichtete Strukturen.

\begin{abstract}
This paper presents a novel procedure for the optimization of band-pass filters consisting of frequency selective surfaces (FSS) embedded in a dielectric. The aim is to optimize the parameters of the complete structure so that the transmission characteristics of the filters fulfill the demanding requirements. The Particle Swarm Optimization (PSO) is used as the optimization procedure. PSO is a new stochastic optimization method that is successfully applied in different areas for the optimization of non-linear problems with several object-functions. In this work, PSO is integrated into the spectral domain analysis for the calculation of the complex FSS structures. The numerical computation is based on the formulation of an integral equation with the help of the spectral Green's function for layered media.
\end{abstract}

\section{Einführung}

Aufgrund von in den letzten Jahren erzielten technologischen Fortschritten ist es heute möglich, frequenzselektive Filter

Correspondence to: Ge Wu (ge.wu@uni-wuppertal.de)

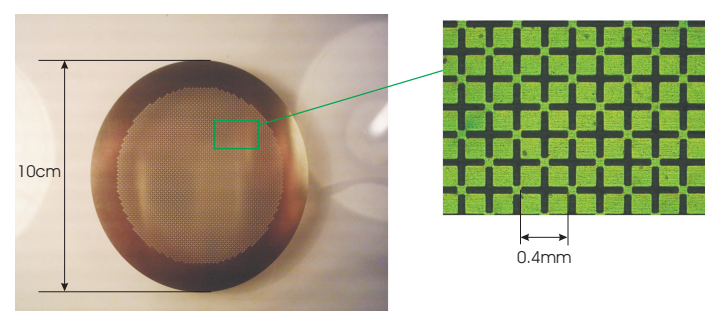

Abbildung 1. Flächenzentriertes Kreuzschlitz-Gitter.

(FSS) aus vielfach geschichteten Strukturen mit komplexen Metallisierungsgeometrien für Anwendungen im Millimeterund Submillimeterwellenbereich herzustellen (Antonopoulos et al., 1997; Hook et al., 2004). So ist es jetzt dem Max-Planck-Institut für Radioastronomie in Bonn gelungen, großflächige induktive FSS-Gitterstrukturen in das Material Polypropylen zu inkorporieren, und zwar so, dass solchermaßen hergestellte Interferenzfilter mechanisch äußerst stabil und gleichzeitig auch noch kryotauglich sind. Damit sind komplex aufgebaute FSS auch in extrem rauscharmen Empfängern, wie sie bei radioastronomischen Experimenten benötigt werden, einsetzbar. Dabei hat sich unter anderem das so genannte flächenzentrierte Kreuzschlitz-Gitter wegen des hervorragenden Resonanzverhaltens durchgesetzt (Wu et al., 2005). Abbildung 1 zeigt ein Foto eines solchen Gitters.

Es wurden im Rahmen dieser Arbeit folgende Anforderungen an Bandpassfilter gestellt (vgl. Abb. 2):

(1) hohe Transmission im Bereich $324-365 G H z$ (für den Bereich $f<324 \mathrm{GHz}$ sind keine hohen Anforderungen notwendig, da die Kante bei $f=324 \mathrm{GHz}$ durch $\mathrm{Zu}-$ sammenschaltung mit einer Hohlleitung scharf abgeschnitten werden kann)

(2) eine Flankensteilheit von $1.05: 1$ bei $f_{\text {Cutoff }}=375 \mathrm{GHz}$

(3) möglichst gute Unterdrückung im Sperrbereich bis $600 \mathrm{GHz}$

Published by Copernicus GmbH on behalf of the URSI Landesausschuss in der Bundesrepublik Deutschland e.V. 


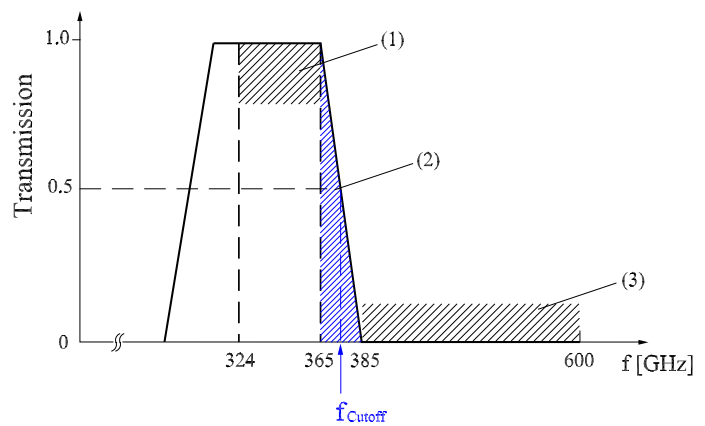

Abbildung 2. Filteranforderungen.
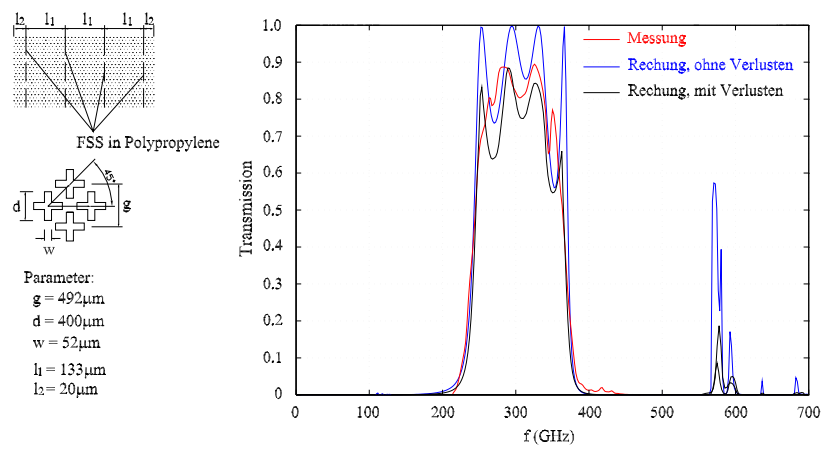

Abbildung 3. Transmissionsfaktor eines 4-lagigen FSS-Bandpassfilters aus flächenzentrierten Kreuzschlitz-Gittern.

Abbildung 3 zeigt das Transmissionsverhalten eines 4lagigen FSS-Bandpassfilters aus einer unserer früheren $\mathrm{Ar}$ beiten. Aus den Transmissionskurven ist ersichtlich, dass die o.g. Filteranforderungen nur teilweise erfüllt worden sind: Wegen der starken Oszillation im Bereich $320-360 \mathrm{GHz}$ ist die erforderliche Transmission im Durchlassbereich nicht erreicht, wodurch die Flankensteilheit ebenfalls negativ beeinflusst wird. Außerdem wird die Ausbreitung höherer Moden im Bereich bis $600 \mathrm{GHz}$ nicht vollständig unterdrückt. Das Syntheseverfahren zur Dimensionierung dieses Filters basierte auf Erfahrungswerten und dem Ersatzschaltbild der Struktur (Wu et al., 2004). Diese Methode hat den Nachteil, dass die Mehrdimensionalität nicht systematisch untersucht werden kann. Das Filter ist allerdings eine Näherung erster Ordnung und sollte deshalb für die weitere Optimierung als Ausgangspunkt genommen werden.

Mit der Integration des Particle Swarm OptimizationAlgorithmus (PSO) in die Berechnungsmethode - die Spektralbereichsanalyse - gelingt es, die Dimensionierung des gesamten Bandpassfilters so zu optimieren, dass das Frequenzverhalten des Filters den gestellten hohen Anforderungen entspricht.

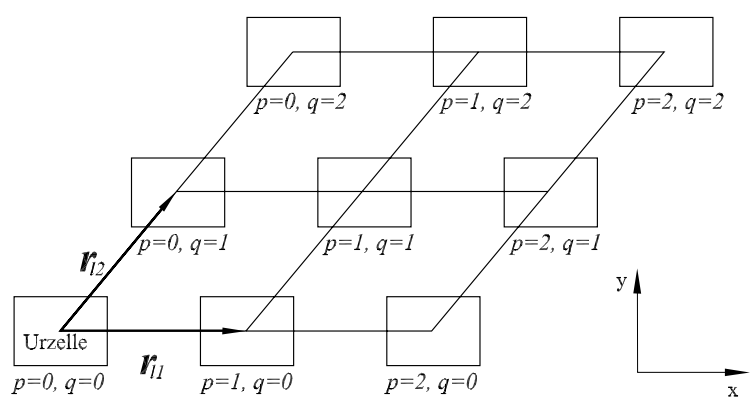

Abbildung 4. Zweidimensionale Verschiebung der Urzelle.

\section{Berechnung von FSS-Strukturen mit Hilfe der Spektralbereichsanalyse}

Das Verfahren zur Berechnung von FSS-Strukturen basiert auf einer Spektralbereichsanalyse, die das feldtheoretische Problem mit Hilfe der Greenschen Funktion für die geschichtete Struktur behandelt. Die Greensche Funktion kann im Spektralbereich in analytischer Form exakt angegeben werden. Das Feldproblem lässt sich als Integralgleichung für den Strombelag auf den metallisierten Strukturen formulieren, die dann mit dem Galerkin-Verfahren der Momentenmethode (MoM) gelöst wird (Aroudaki et al., 1995). Abb. 4 stellt ein Modell einer allgemeinen periodischen Gitterstruktur als 2dimensionale Verschiebungen der so genannten Urzelle mit Hilfe von zwei Gittervektoren dar:

$\boldsymbol{r}_{l p q}=p \boldsymbol{r}_{l 1}+q \boldsymbol{r}_{l 2}$

Bei den induktiven Strukturen sind die Grundelemente Aperturen, die mit äquivalenten magnetischen Oberflächenstromdichten belegt werden.

Mit Hilfe des Floquet-Theorems lässt sich die gesamte Stromdichte als periodische Fortsetzung der Stromdichte in der Urzelle mit entsprechenden Phasenverschiebungen darstellen. Die äquivalente magnetische Stromdichte in einer Ebene und die entsprechende Fouriertransformierte im Spektralbereich lauten:

$$
\begin{aligned}
& \boldsymbol{M}(x, y, z)=\sum_{p=-\infty}^{p=+\infty} \sum_{q=-\infty}^{q=+\infty} \sum_{n=1}^{N_{l}} U_{l n} \\
& \boldsymbol{f}_{l n}\left(x-\Delta x_{l p q}, y-\Delta y_{l p q}\right) \cdot e^{j\left(k_{e x} \Delta x_{l p q}+k_{e y} \Delta y_{l p q}\right)}
\end{aligned}
$$

und

$$
\begin{gathered}
\boldsymbol{M}\left(k_{x}, k_{y}\right)=4 \pi^{2} \cdot \sum_{n=1}^{N_{l}} U_{l n} \cdot \boldsymbol{F}_{l n}\left(k_{x}, k_{y}\right) \\
\cdot \sum_{p=-\infty}^{p=+\infty} \delta\left[\left(k_{x}-k_{e x}\right) r_{l 1 x}+\left(k_{y}-k_{e y}\right) r_{l 1 y}-2 \pi p\right] \\
\quad \cdot \sum_{q=-\infty}^{q=+\infty} \delta\left[\left(k_{x}-k_{e x}\right) r_{l 2 x}+\left(k_{y}-k_{e y}\right) r_{l 2 y}-2 \pi q\right],
\end{gathered}
$$


wobei $\boldsymbol{f}_{\ln }(x, y)$ und $\boldsymbol{F}_{l n}\left(k_{x}, k_{y}\right)$ die Basisfunktionen, $k_{e x}$ und $k_{e y}$ die Wellenzahlen des einfallenden Feldes und $U_{n}$ die unbekannten Amplituden der magnetischen Stromdichte sind. Das H-Feld, erzeugt durch die Strommoden auf dieser Ebene, gewinnt man durch eine Rücktransformation der Raumfrequenzbereichsformulierung in den Ortsbereich:

$$
\begin{aligned}
& \boldsymbol{H}(x, y, z)=\iint_{-\infty}^{\infty} \stackrel{\leftrightarrow}{G}_{M}^{H}\left(k_{x}\left(k_{x}^{\prime}, k_{y}^{\prime}\right), k_{y}\left(k_{x}^{\prime}, k_{y}^{\prime}\right), z, z^{\prime}=z_{l}\right) \\
& \cdot \boldsymbol{M}_{l}\left(k_{x}^{\prime}, k_{y}^{\prime}\right) e^{j\left(k_{x}\left(k_{x}^{\prime}, k_{y}^{\prime}\right) x+k_{y}\left(k_{x}^{\prime}, k_{y}^{\prime}\right) y\right)} \\
& \cdot\left|\frac{\partial\left(k_{x}, k_{y}\right)}{\partial\left(k_{x}^{\prime}, k_{y}^{\prime}\right)}\right| d k_{x}^{\prime} d k_{y}^{\prime}
\end{aligned}
$$

Es folgt

$\boldsymbol{H}(x, y, z)=\frac{1}{\left|r_{l 1 x} \cdot r_{l 2 y}-r_{l 2 x} \cdot r_{l 1 y}\right|}$

$\cdot \sum_{n=1}^{N_{l}} \sum_{p=-\infty}^{\infty} \sum_{q=-\infty}^{\infty} U_{l n} \cdot \stackrel{\leftrightarrow}{G}_{M}^{H}\left(k_{x}(l, p, q), k_{y}(l, p, q), z, z^{\prime}=z_{l}\right)$

$\cdot \boldsymbol{F}_{l n}\left(k_{x}(l, p, q), k_{y}(l, p, q)\right) \cdot e^{j\left(k_{x}(l, p, q) x+k_{y}(l, p, q) y\right)}$,

$\leftrightarrow H$

$\stackrel{\leftrightarrow}{G}_{M}$ ist die spektrale Greensche Funktion für das magnetische Feld, $k_{x}(l, p, q)$ und $k_{y}(l, p, q)$ sind die diskreten Wellenzahlen

$$
\begin{aligned}
& k_{x}(l, p, q)=k_{e x}+\frac{2 \pi}{\left|r_{l 1 x} \cdot r_{l 2 y}-r_{l 2 x} \cdot r_{l 1 y}\right|} \\
& \cdot\left(r_{l 2 y} \cdot p-r_{l 1 y} \cdot q\right) \\
& k_{y}(l, p, q)=k_{e y}-\frac{2 \pi}{\left|r_{l 1 x} \cdot r_{l 2 y}-r_{l 2 x} \cdot r_{l 1 y}\right|} \\
& \cdot\left(r_{l 2 x} \cdot p-r_{l 1 x} \cdot q\right) .
\end{aligned}
$$

Durch Erfüllung der entsprechenden Stetigkeitsbedingungen des H-Feldes gewinnt man die Systemmatrix in der Form

$$
\begin{gathered}
Z_{m n}\left(l, l^{\prime}\right)=\frac{1}{\sum_{p=-\infty}^{\infty} \sum_{q=-\infty}^{\infty}{\stackrel{\leftrightarrow}{r_{l^{\prime} 1 x} \cdot r_{l^{\prime} 2 y}-r_{l^{\prime} 2 x} \cdot r_{l^{\prime} 1 y} \mid}}_{M}\left(k_{x}\left(l^{\prime}, p, q\right), k_{y}\left(l^{\prime}, p, q\right), z=z_{l}, z^{\prime}=z_{l^{\prime}}\right)} \\
\cdot \boldsymbol{F}_{l^{\prime} n}\left(k_{x}\left(l^{\prime}, p, q\right), k_{y}\left(l^{\prime}, p, q\right)\right) \\
\cdot \boldsymbol{F}_{l m}\left(-k_{x}(l, p, q),-k_{y}(l, p, q)\right) .
\end{gathered}
$$

Der Vorteil dieses Verfahrens besteht darin, dass das Feld ohne Lösen des Integrals in exakter Form angegeben werden kann. Auf der Basis dieser Theorie wurde ein Computerprogramm an unserem Lehrstuhl entwickelt, und die Berechnungen zahlreicher FSS-Strukturen wiesen gute bereinstimmung mit Messwerten auf (Aroudaki, 1996).

Bei der numerischen Simulation können auch die Verluste im Dielektrikum berücksichtigt werden. Da diese nicht in einem direkten Zusammenhang mit der Optimierung der Strukturen stehen, werden sie bei der Anwendung des Optimierungsverfahrens zunächst vernachlässigt.

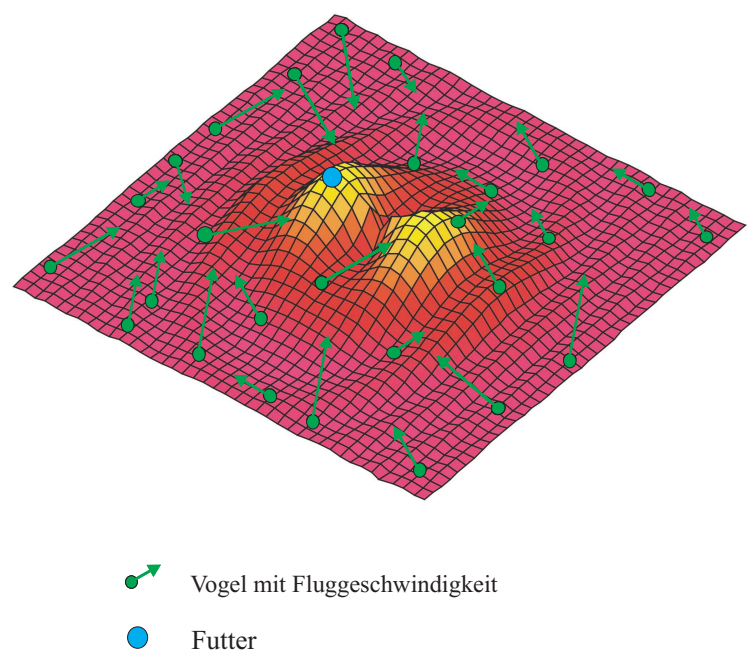

Abbildung 5. Gruppe von gleichmäßig verteilten Vögeln auf der Suche nach Futter.

\section{PSO-Algorithmus}

Particle Swarm Optimization (PSO) ist ein stochastisches Optimierungsverfahren, das auf einem evolutionären Algorithmus basiert. Das Verfahren hat seinen Ursprung in Simulationsmodellen zur Visualisierung des Sozialverhaltens von Vogelschwärmen. Die grundlegenden berlegungen stammen von Kennedy und Eberhart (Kennedy and Eberhart, 1995).

Ein mögliches Szenario (siehe Abb. 5) ist eine Gruppe von gleichmäßig verteilten Vögeln, die auf der Suche nach Futter sind. Das Futter befindet sich an einem einzigen Platz, die Vögel suchen danach und benutzen dabei die schon gesammelten Informationen. Bei der Aktualisierung der Positionen der einzelnen Vögel wird bei jedem Zeitschritt nach dem Optimum (hier das Futter) gesucht, bis alle Vögel das Futter finden und sich in der Nähe des Futters aufhalten, mit anderen Worten: das Optimierungsproblem konvergiert.

In der PSO wird jeder Vogel als ein Partikel bezeichnet, der folgende Eigenschaften aufweist:

- Jedem Partikel wird ein Fitness-Wert zur Beurteilung der aktuellen Position zugewiesen. Der Fitness-Wert wird mit Hilfe einer problemangepassten Funktion, der Fitness-Funktion, bestimmt.

- Jedes Partikel hat ein Gedächtnis, und seine bisherige beste Position und deren Fitness-Wert werden gespeichert. Diese Position nennt sich pbest (particle best solution). Zusätzlich erfährt das Partikel die beste Position des gesamten Schwarms - die so genannte gbest (globel best solution).

- Bei jeder Festlegung der Bewegung ist das Partikel bereit, die beiden Positionen - pbest und gbest - zu berücksichtigen bzw. zu verfolgen. 


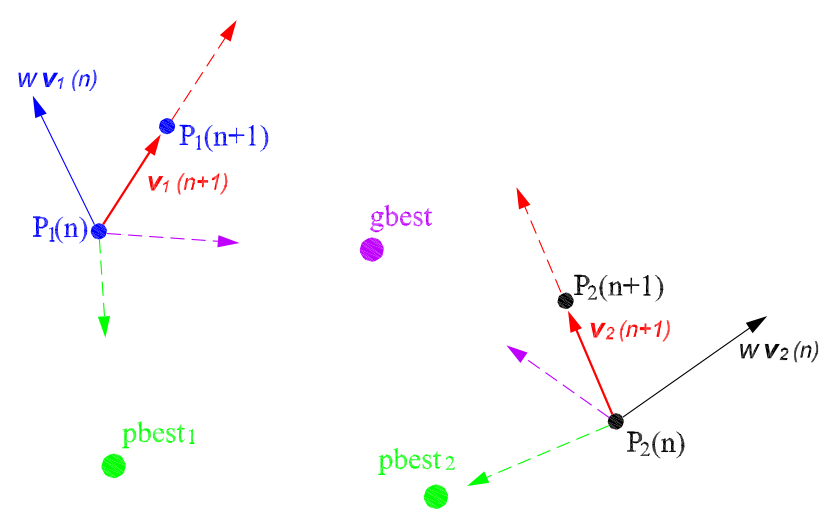

Abbildung 6. 2-D-Darstellung der Geschwindigkeitsberechnung.

- Jedes Partikel besitzt eine Geschwindigkeit zur Steuerung der nächsten Bewegung.

Das Update der Geschwindigkeit und der Position erfolgt nach den Gleichungen

$$
\begin{aligned}
\boldsymbol{v}_{i, n+1}=w * \boldsymbol{v}_{i, n} & +c_{1} \operatorname{rand}() *\left(\boldsymbol{p}_{\text {best } i, n}-\boldsymbol{x}_{i, n}\right) \\
& +c_{2} \operatorname{rand}() *\left(\boldsymbol{g}_{\text {best } n}-\boldsymbol{x}_{i, n}\right)
\end{aligned}
$$

und

$\boldsymbol{x}_{i, n+1}=\boldsymbol{x}_{i, n}+\boldsymbol{v}_{i, n+1} * \Delta t$.

Die Vektoren $\boldsymbol{v}$ und $\boldsymbol{x}$ repräsentieren jeweils die Geschwindigkeit und die Position eines $i$-ten Partikels im mehrdimensionalen Lösungsraum. Der Index $n$ steht für den $n$-ten Iterationsschritt, $n+1$ dann für den nächsten Iterationsschritt. rand( ) ist eine zufällige Zahl zwischen 0 und 1. Die Vektoren $\boldsymbol{p}_{\text {best }}$ und $\boldsymbol{g}_{\text {best }}$ sind jeweils die Positionen der bisherigen eigenen und der globalen besten Lösungen. $\Delta t$ ist der diskrete Zeitschritt. Laut Gl. (9) gibt es 3 Steuerparameter zum Update der Geschwindigkeit: $w, c_{1}$ und $c_{2}$. $w$ wird Dämpfungsgewicht genannt. Es kontrolliert die Auswirkung der vorherigen Geschwindigkeit auf die neue Geschwindigkeit und damit die Abstimmung zwischen der globalen und der lokalen Suche. Ein großer Wert für $w$ unterstützt vor allem die globale, ein kleiner die lokale Suche. $c_{1}$ und $c_{2}$ sind die so genannten Lern-Konstanten, die dafür sorgen, wie weit die momentane Geschwindigkeit durch pbest und gbest verändert wird, sie beschreiben also den Einfluss eigener und anderer Erfahrungen. Durch geschickte Wahl der PSO-Parameter kann die durchschnittliche Anzahl der Iterationen gesenkt werden, bis ein Optimum gefunden wird.

Abbildung 6 zeigt eine 2-D-Darstellung der Geschwindigkeitsberechnung. Die ursprüngliche Geschwindigkeit des Partikels Nr. 1, also $\boldsymbol{v}_{1}(n)$, wird sowohl von pbest 1 als auch von gbest abgelenkt und bewegt sich nach der resultierenden Geschwindigkeit $\boldsymbol{v}_{1}(n+1)$. Diese ist nun die Geschwindigkeit im nächsten Zeitschritt.
Insgesamt lässt sich der Pseudocode für PSO folgendermaßen schreiben:

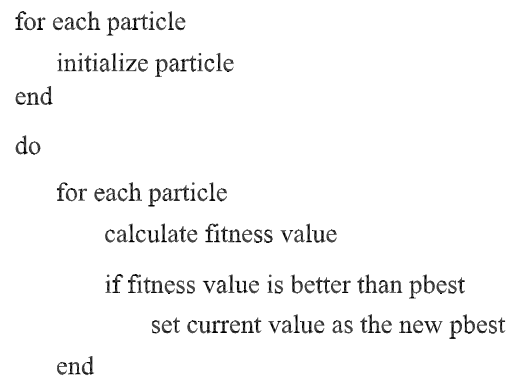

Der erste Schritt ist die Initialisierung der Startposition und der Geschwindigkeit jedes Partikels. Diese erfolgt durch einen Zufallsgenerator, so dass alle Partikel gleichmäßig im gesamten Lösungsraum verteilt sind. Im zweiten Schritt wird die aktuelle Position durch die Fitness-Funktion bewertet und für jedes Partikel wird ein pbest gefunden. Der nächste Schritt ist die Wahl bzw. die Aktualisierung der besten Position gbest des gesamten Schwarms. Dann folgen das Update der Geschwindigkeit nach Gl. (9) und das Update der Position nach Gl. (10). Anschließend springt der Algorithmus zurück zum Schritt 2, bis die maximale Iteration erreicht wird oder das letzte gbest ein zufriedenstellendes Ergebnis liefert. gbest ist nach jedem Durchlauf der Schleife die beste Position des gesamten Schwarms in allen bisherigen Zeitschritten, und gbest des letzten Zeitschritts repräsentiert also die optimierte Lösung des Problems.

In den letzten Jahren hat sich PSO als ein effizientes Verfahren für die Optimierung sehr unterschiedlicher komplexer Problemstellungen durchgesetzt (Eberhart and Shi, 2001; Robinson and Rahmat-Samii, 2004). Das Verfahren ist besonders geeignet für die Lösung von nicht linearen, multidimensionalen Optimierungsproblemen mit mehreren Zielfunktionen. Vor allem wegen ihrer schnellen Konvergenz hat sich PSO als eine effiziente Methode für die Optimierung der Parameter unserer FSS-Filterstrukturen erwiesen.

\section{Integration von PSO in die Berechnung der FSS-Bandpassfilter}

Die Integration von PSO in die Berechnung der FSSBandpassfilter erfolgt in 30 Schritten.

Im ersten Schritt wird der Lösungsraum definiert. In Abb. 7 ist ein 4-lagiges FSS-Bandpassfilter schematisch dar- 

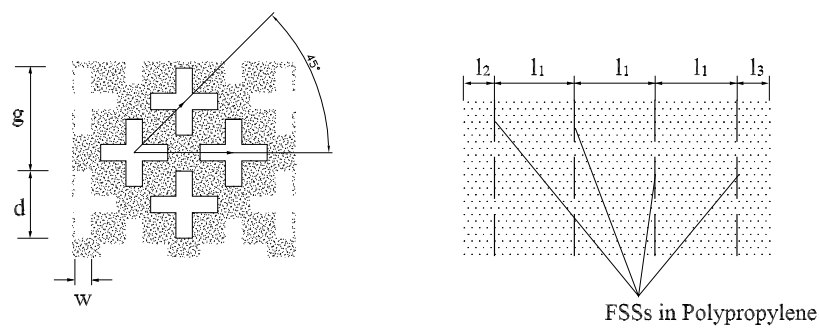

Abbildung 7. Schematische Darstellung eines 4-lagigen FSSBandpassfilters.

gestellt. Die zu optimierenden Parameter sind die Gitterparameter $g, d$, und $w$ und die Schichtdicken $l_{1}, l_{2}$ und $l_{3}$, so dass ein 6-dimensionales Optimierungsproblem entsteht. Die Grenzen des Lösungsraums werden aus technologischen Gründen für jede Dimension wie folgt festgelegt:

$g \in(380,480) ; d \in(310,370) ; w \in(40,140) ;$

$l_{1} \in(80,170) ; l_{2} \in(0,60) ; l_{3} \in(0,60)$.

Bei der Wahl der Grenzwerte wurden die Erfahrungswerte einbezogen. Neben dem 4-lagigen werden auch 3-lagige Filter untersucht. Da wir uns - ebenfalls aus technologischen Gründen - auf symmetrische Filter beschränken, d.h. die Zwischenschichtdicken sind alle gleich $\left(=l_{1}\right)$, handelt es sich bei den 3-lagigen Filtern ebenfalls um ein 6dimensionales Optimierungsproblem.

Der zweite Schritt ist die Formulierung der FitnessFunktion. Bezogen auf die Filteranforderungen (siehe Abb. 2) wird die Fitness-Funktion als

$f(\boldsymbol{x})=w_{1} f_{1}(\boldsymbol{x})+w_{2} f_{2}(\boldsymbol{x})+w_{3} f_{3}(\boldsymbol{x})$

definiert, die aus drei Teilfunktionen

$\begin{array}{ll}f_{1}(\boldsymbol{x})=\frac{1}{n} \sum_{324 G}^{365 G} T^{2}, & w_{1}=0.4 \\ f_{2}(\boldsymbol{x})=T_{365 G}^{2}-T_{385 G}^{2}, & w_{2}=0.4\end{array}$

und

$f_{3}(\boldsymbol{x})=\frac{1}{n} \sum_{385 G}^{600 G}\left(1-T^{2}\right) \quad w_{3}=0.2$

besteht, die jeweils die Filteranforderungen für verschiedene Frequenzbereiche mathematisch beschreiben: $f_{1}(\boldsymbol{x})$ für den Durchlassbereich, $f_{2}(\boldsymbol{x})$ für die Flankensteilheit und $f_{3}(\boldsymbol{x})$ für den Sperrbereich. $n$ ist die Anzahl der berechneten Frequenzpunkte, $w_{1}, w_{2}$ und $w_{3}$ sind die Gewichtungen der jeweiligen Teilfunktionen. Nach dieser Festlegung besitzt die gesamte Fitness-Funktion $f(\boldsymbol{x})$ einen Wert zwischen 0 und 1.

Im letzten Schritt werden die PSO-Parameter bestimmt. Das Dämpfungsgewicht soll nach der Gleichung

$w(n)=w_{o}-\left(w_{o}-w_{u}\right) \frac{n}{\text { MaxIter. }}$

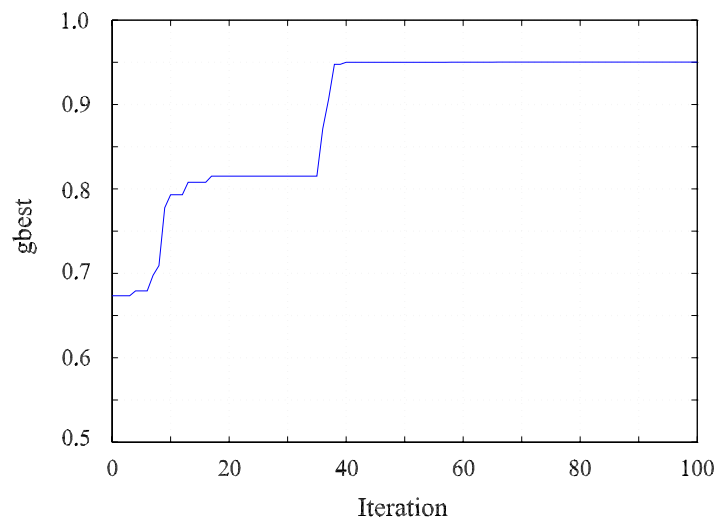

Abbildung 8. Konvergenzverhalten des besten Fitness-Wertes (4lagiges Filter, Anzahl der Partikel = 50).

mit

$w_{o}=0.91$ und $w_{u}=0.28$

einen linearen Abstieg vom Anfangswert 0.9 bis zum Endwert 0.28 bezüglich der Iterationsschritte bewerten, so dass am Anfang eine globale, und am Ende eine überwiegend lokale Suche stattfindet.

Beide Lern-Konstanten $c_{1}$ und $c_{2}$ werden erfahrungsgemäß gleich 2.0 gesetzt.

Es ist noch notwendig, eine maximale Geschwindigkeit $\boldsymbol{v}_{\text {max }}$ für die Beschränkung der Bewegung zu definieren, d.h., wenn die Geschwindigkeit eines Partikels $\boldsymbol{v}_{\text {max }}$ überschreitet, wird sie gleich $\boldsymbol{v}_{\max }$ gesetzt. Wir halbieren die Grenzen des Lösungsraums zur Festlegung von $\boldsymbol{v}_{\max }$ :

$$
\begin{aligned}
\boldsymbol{v}_{\max }\left(g, d, w, l_{1}, l_{2}, l_{3}\right) & =0.5\left(\boldsymbol{x}_{\max }-\boldsymbol{x}_{\min }\right) \\
& =(50,30,50,45,30,30)
\end{aligned}
$$

\section{Numerische Ergebnisse}

Im Folgenden werden die numerischen Ergebnisse präsentiert.

Abbildung 8 zeigt das Konvergenzverhalten von gbest für ein 4-lagiges Filter. Nach etwa 40 Iterationen konvergiert gbest zu einem hohen Fitness-Wert von 0.95. Der entsprechende optimale Lösungsverktor ist

$$
\begin{array}{r}
\boldsymbol{g}_{\text {best }}\left(g, d, w, l_{1}, l_{2}, l_{3}\right) \\
=(451.9,387.2,50.1,157.0,20.1,20.3)
\end{array}
$$

In Abb. 9 ist der Transmissionsfaktor dieses optimierten Filters dargestellt. Die Kurve des nicht optimierten aus Abb. 3 ist zum Vergleich ebenfalls aufgetragen. Aus den Kurven kann man schließen, dass das optimierte 4-lagige Filter in allen 3 Teilfrequenzbereichen die Anforderungen erfüllt (die Flankensteilheit errechnet sich zu 1.056:1). Der 

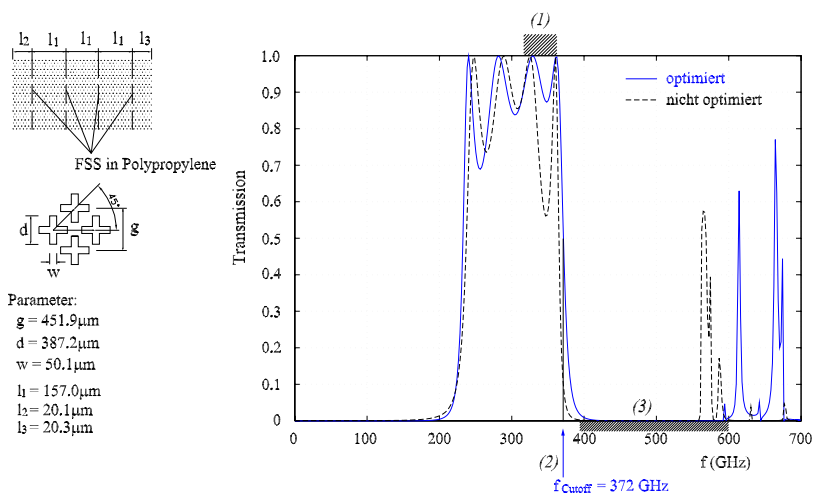

Abbildung 9. Transmissionsfaktor des optimierten 4-lagigen Filters.

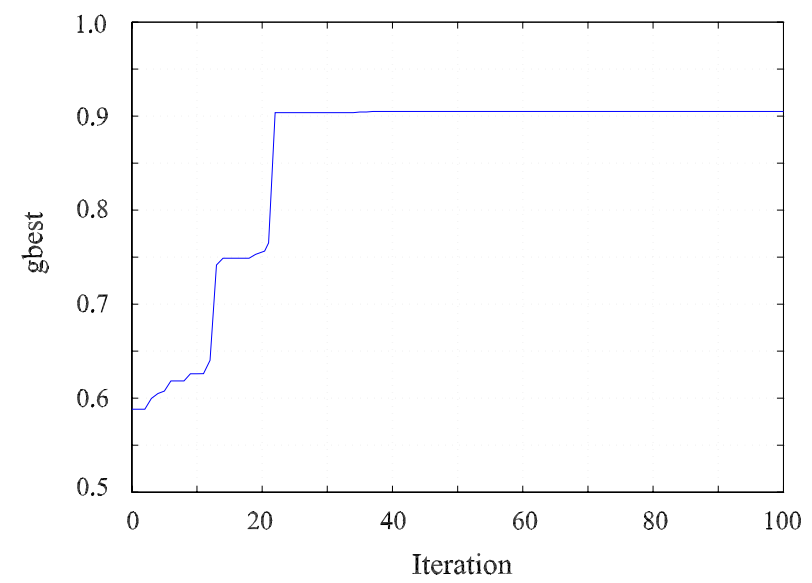

Abbildung 10. Konvergenzverhalten des besten Fitness-Wertes (3-lagiges Filter, Anzahl der Partikel = 50).

hohe Fitness-Wert wird durch die Transmissionseigenschaften bestätigt.

Abbildungen 10 und 11 stellen die Ergebnisse eines 3lagigen Filters mit

$$
\begin{aligned}
\boldsymbol{g}_{\text {best }}\left(g, d, w, l_{1}, l_{2}, l_{3}\right) \\
\quad=(474.2,352.3,59.2,159.2,24.4,24.3)
\end{aligned}
$$

dar.

Mit der gleichen Anzahl der Partikel konvergiert der Optimierungsprozess zwar schneller als der des 4-lagigen Filters, es wird aber nur ein kleinerer Fitness-Wert von 0.9 erreicht. Die Filteranforderungen werden teilweise gut erfüllt. In den Bereichen (1) und (3) liefert das Filter gute Ergebnisse. Dagegen wird im Bereich (2) eine Flankensteilheit von 1.05:1 nicht erreicht.
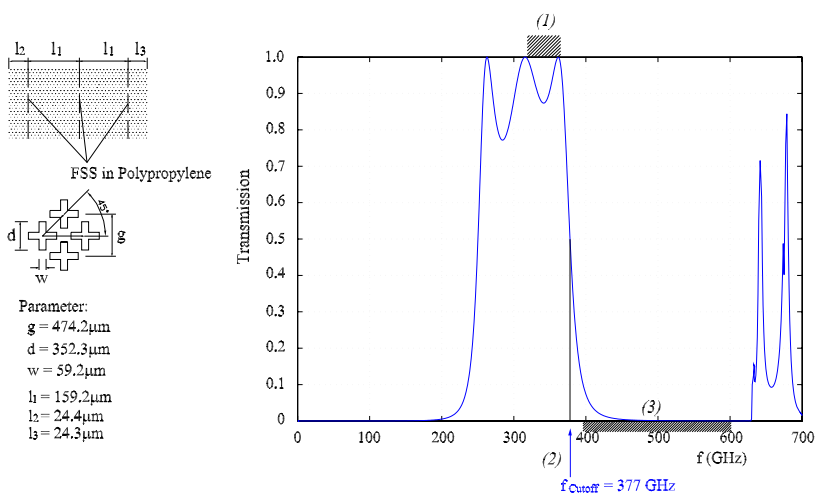

Abbildung 11. Transmissionsfaktor des optimierten 3-lagigen Filters.

\section{Zusammenfassung}

Diese Arbeit befasst sich mit der Optimierung von FSSBandpassfiltern für Anwendungen in der Radioastronomie mit strengen Anforderungen an die Filtereigenschaften. Als Geometrie für die Metallisierungen werden flächenzentrierte Kreuzschlitzelemente gewählt.

Die exakte Berechnung der Struktur erfolgt mit der Spektralbereichsanalyse, in die der PSO-Algorithmus integriert wird. Durch Definitionen des Lösungsraums und der FitnessFunktion und durch die Festlegung der PSO-Parameter wird der Optimierungsalgorithmus an die FSS-Filterstrukturen angepasst. Zwei optimierte Filter - ein 4-lagiges und ein 3lagiges Filter - werden präsentiert. Das 4-lagige Filter zeigt bessere Transmissionscharakteristiken. Für die praktische Anwendungen sind noch die Verluste sowohl im Metall als auch im Dielektrikum zu berücksichtigen; deshalb wird versucht, die Anzahl der Schichten gering zu halten. Aus diesem Grund ist das 3-lagige Filter ebenfalls interessant, wenn man einige Abweichungen von den Filteranforderungen akzeptiert.

Die hier vorgestellte Vorgehensweise hat eine allgemeine Bedeutung bezüglich sehr unterschiedlicher Anwendungsgebiete. So ist die Optimierung weiterer Strukturen mit unterschiedlichen Zielfunktionen möglich.

\section{Literatur}

Antonopoulos, C., Parker, E. A., and Sturland, I. M.: Multilayer frequency selective surfaces for millimetre and submillimetre wave applications, Proc. IEE Microwave Antennas and Propagation, 144, 415-420, 1997.

Aroudaki, H., Hansen, V., Gemünd, H., and Kreysa, E.: Analysis of low-pass filters consisting of multiple stacked FSS's of different periodicities with applications in the submillimeter radioastronomy, IEEE Trans. Antennas Propagat., 43, 1486-1491, 1995. 
Aroudaki, H.: Spektralbereichsanalyse von mehrlagigen planaren Schaltungen mit dreidimensionalen Diskontinuitäten, Diss. Bergische Univ. Wuppertal, 1996.

Eberhart, R. and Shi, Y.: Particle swarm optimization: development, application and resources, Proc. 2001 Congr. Evolutionary Computation, Seoul, Korea, 1, 2001.

Hook, M., Vardaxoglou, J. C., and Ward, K. Applications of Frequency Selective Surfaces, 27th ESA Antenna Workshop on Innovative Periodic Antennas, Santiago de Compostella, Spain, 299-309, 2004.

Kennedy, J. and Eberhart, R.: Particle Swarm Optimization, Proc. of the IEEE Intern. Conf. on Neural Networks, Piscataway, NJ:IEEE Press, 1942-1948, 1995.
Robinson, J. and Rahmat-Samii, Y.: Particle Swarm Optimization in Electromagnetics, IEEE Transactions on Antennas and Propagation, 52, 397-407, 2004.

Wu, G., Hansen, V., Gemuend, H.-P., and Kreysa, E.: Multi-layered Submillimetre FSS of Shifted Crossed Slot Elements for Applications in Radio Astronomy, German Microwave Conf., Ulm, Germany, 2005.

Wu, G., Hansen, V., Kreysa, E., and Gemuend, H.-P.: Synthesis of High-Grade Filters for TeraHertz Applications Based on Multilayered Structures Using Capacitive and Inductive Grids, 27th ESA Antenna Workshop on Innovative Periodic Antennas, Santiago de Compostella, Spain, 321-327, 2004. 